\title{
TOLERABILITY OF TOPICAL NSAIDS IN THE ELDERLY:
}

\section{DO THEY REALLY CONVEY A SAFETY ADVANTAGE?}

\author{
Josie MM Evans BA(Oxon) \\ Research Assistant \\ Thomas M MacDonald MD FRCP(Edin) \\ Senior Lecturer / Consultant Physician \\ Medicines Monitoring Unit \\ Department of Clinical Pharmacology \\ University of Dundee
}

Running title: Safety of topical NSAIDs in the elderly 


\section{Acknowledgements}

The Medicines Monitoring Unit is supported by a grant from the Medicines Control Agency. 
Name and address for correspondence

Dr TM MacDonald

Medicines Monitoring Unit

Department of Clinical Pharmacology

Ninewells Hospital \& Medical School

Dundee DD1 9SY

Phone: 01382-660111 Ext 3405

Fax: 01382-644972 


\section{SUMMARY}

Non-steroidal anti-inflammatory drugs (NSAIDs) are very widely prescribed but they have a poor safety profile, with a range of potential adverse effects. NSAIDs that are used in topical formulation have been developed in the last fifteen years and their use is increasing. The purpose is to achieve a high local concentration of the active ingredient at the affected site, with as low a plasma concentration as possible to minimise possible systemic side-effects. This paper reviews the evidence available regarding the safety of topical NSAIDs, with particular reference to gastrointestinal and renal toxicity which have recently been highlighted in the medical press. The effects on the elderly are also discussed. The authors conclude that more formal epidemiological evaluation of the safety of topical NSAIDs is required in the post-marketing situation. 
Non-steroidal anti-inflammatory drugs (NSAIDs) are among the most commonly prescribed drugs world-wide. There are about 20 million prescriptions dispensed per year in the UK at a 1993 cost of over $£ 180 \mathrm{~m}\{1\}$. In Tayside, Scotland, which has a population of nearly 400 thousand people, 150 thousand patients received 736 thousand prescriptions for NSAIDs in the five year period from 1989 to 1993 . Over one quarter of the patients receiving NSAID prescriptions were elderly, and they recived almost one half of the NSAID prescriptions (Table 1). The prevalence of NSAID use was calculated by determining the percentage of the population who dispensed at least one NSAID between 1989 and 1993, using mid-1991 population estimates $\{1083\}$. This was nearly $60 \%$ in the elderly.

That NSAIDs are useful in the treatment of rheumatoid arthritis and other inflammatory arthropathies is beyond doubt $\{399\}$, but they are frequently associated with serious or life threatening adverse reactions and are responsible for a quarter of all serious adverse drug reactions reported to the Committee on Safety of Medicines (CSM) on Yellow Cards $\{615\}$. Indeed, enteral NSAIDs appear to be associated with toxicity of similar magnitude to secondline drugs $\{1812\}$.

\section{SIDE-EFFECTS OF ORAL NSAIDS}

The main adverse drug reactions occurring with NSAIDs, with particular reference to the elderly, have been described in a recent review $\{1771\}$.

\section{Gastrointestinal toxicity}


In broader populations, the toxicity of NSAIDs on the upper $\{498\}\{136\}\{466\}\{1397\}\{129\}$ and lower $\{1137\}\{518\}\{401\}$ gastrointestinal tract is well documented. Such gastrointestinal mucosal damage is the result of the inhibition of prostaglandin synthesis $\{517\}$. Patients exposed to NSAIDs therefore have an increased risk of serious complications from peptic ulcer disease. The incidence of NSAID-induced erosions and concomitant haemorrhage and perforation ranges from 6,000 to 20,000 each year in the $\mathrm{UK}\{1390\}$. Increasing age increases both the relative and absolute risk of NSAID-induced upper gastrointestinal damage $\{554\}\{517\}$ because elderly patients already have a higher background risk $\{517\}$.

\section{Renal side-effects}

NSAIDs may also cause renal impairment leading to acute and chronic renal failure $\{1008\}$. Although earlier pharmacoepidemiological studies failed to show any risk or showed that the risk was low $\{1015\}$, evidence is now available from randomised controlled studies $\{1011\}$ and observational studies $\{1107\}$. In addition, a recent study carried out using an automated database at the Medicines Monitoring Unit, University of Dundee (MEMO) $\{1766\}$ found an approximate doubling of the risk of hospitalisation for acute renal failure with the use of NSAIDs $\{1401\}$. The elderly appear more susceptible to renal toxicity, probably due to reduced renal reserve $\{1108\}$.

\section{Hepatotoxicity}

NSAID-induced hepatotoxicity is a well-recognised but rare adverse effect $\{1729\}$. One recent cohort study found that current NSAID users have twice the background risk of newly diagnosed acute liver injury $\{544\}$. In this study, the rate ratio was the same across all age 
groups. However, NSAID induced liver damage is more common among elderly patients, because they have a higher background rate and are more likely to be exposed to NSAIDs. For example, among 70 cases of acute liver disorder $\{1729\}$, a causal effect of NSAIDs could not be ruled out for nine. Of these nine, six were over the age of 60 years.

\section{Other effects}

NSAIDs raise blood pressure $\{1148\}$ and their use results in increased prescribing of antihypertensive drugs $\{1146\}$. They have haematological effects, notably decreased platelet adhesiveness and increased risk of bleeding $\{4\}$. NSAIDs can also cause hypersensitivity reactions and rashes $\{4\}$, and worsening of osteoarthritis $\{1809\}$. They may also be toxic to the bone marrow $\{1811\}$. Since 1975, the CSM has been aware that NSAIDs can cause bronchospasm in susceptible patients $\{100\}$, and the British National Formulary contains a CSM warning that any degree of worsening of asthma may be associated with NSAID ingestion $\{762\}$. As with all drugs, the possibility of NSAIDs causing confusion in the elderly should also be considered $\{1771\}$.

\section{Interactions}

There are many NSAID-drug interactions $\{101\}$, in which the NSAID is often the activating agent $\{1771\}$. This is a particular problem in the elderly, partly as a result of polypharmacy, and they require close monitoring.

There is now good evidence that NSAID toxicity is dose related, and that some NSAIDs are more toxic than others $\{136\}\{436\}\{1359\}$. 


\section{STRATEGIES FOR REDUCING NSAID TOXICITY}

The toxicity of NSAIDs could be reduced in several ways. First, it has been suggested that many patients with osteoarthritis could be managed with simple analgesics rather than NSAIDs $\{1773\}\{1813\}\{1829\}$. Presumably such advice could be extended to include the symptomatic treatment of simple musculoskeletal symptoms where an anti-inflammatory action is not required.

Second, where an NSAID is indicated, the lowest effective doses of the least toxic NSAIDs should be used $\{1350\}$.

There are some patients at high risk from NSAID toxicity but for whom no other therapy can be given. These include the elderly and those either with symptoms or a history of an upper gastrointestinal disorder. In such cases prophylactic therapy with prostaglandin analogues, shown to reduce serious upper gastrointestinal complications $\{1810\}$, or other ulcer healing drugs, not yet shown unequivocally to reduce upper gastrointestinal haemorrhage $\{506\}$, should be used.

These strategies apply equally to patients receiving NSAIDs for the first time and those receiving chronic prescriptions, as the risk from NSAIDs is probably constant with continuous exposure $\{1669\}$.

\section{Topical NSAIDs}


Another alternative is to prescribe NSAIDs in topical formulation. The development, over the last fifteen years, of NSAID preparations that are administered topically has been welcomed from a safety standpoint. Fig 1 shows that use of topical NSAIDs has increased in Tayside in the elderly population between 1989 and 1993. Table 2 shows utilisation and prevalence by age and sex for the same time period, and it is clear that although topical NSAIDs are used less commonly than oral NSAIDs, there is still the same pattern of increased use in the elderly.

The rationale behind using NSAIDs in topical formulation is to achieve a high local concentration of the active ingredient at the affected site, with as low a plasma concentration as possible in order to minimise systemic side-effects $\{1768\}$. The short distance of transmission from the site of application (the skin) to the target (the joint), and the avoidance of the entero-hepatic cycle, is an attractive concept. While first generation topical NSAIDs achieved plasma levels of $5-10 \%$ of those reached with oral NSAIDs, this figure is less than $2 \%$ for some of the second generation topicals $\{1769\}$. Although some studies have shown that topical NSAIDs are more effective than placebo at providing relief for a range of indications $\{1242\}\{1248\}\{1776\}\{1247\}\{598\}$, they have not been extensively tested against oral analgesics $\{602\}$. There is widespread, if not substantiated, opinion that topical NSAIDs are clinically less effective than oral NSAIDs $\{1350\}\{1768\}$. However, it is the safety of topical NSAIDs rather than the efficacy, particularly in the elderly, that is the topic of this article. This is becoming progressively more important as the use of topical NSAIDs increases. To date, no topical NSAID has had its license withdrawn due to serious sideeffects, in contrast to the oral NSAID experience $\{334\}\{335\}\{1814\}$.

\section{ADVERSE EFFECTS OF TOPICAL NSAIDS}




\section{Dermatological effects}

Local skin sensitivity, contact dermatitis and photodermatitis are well recognised adverse effects of topical NSAIDs. These are common enough to be detected in small randomised clinical trials. A review of four topical NSAIDs found the highest reported incidence of such skin reactions was $2.6 \%\{3\}$. It is therefore advised that they are not used on broken or inflamed skin $\{762\}$.

\section{Systemic Side-Effects}

One important question is whether or not the plasma concentrations produced by topical NSAIDs are high enough to cause systemic side-effects of the same nature as those of oral NSAIDs. The British National Formulary contains a caution that topical application of large amounts of these drugs may result in systemic side-effects, including hypersensitivity and asthma $\{762\}$. They are thus contraindicated in patients with known hypersensitivity to NSAIDs $\{1768\}\{468\}$.

Several investigators have reported either a low or no incidence of systemic side-effects in randomised controlled trials of patients exposed to topical NSAIDs. It should be pointed out, however, that these studies involved small numbers of patients and elderly patients were excluded $\{1077\}\{1242\}\{1248\}\{1776\}\{602\}\{1247\}$. In a post-marketing surveillance study of 23,590 patients exposed to felbinac $5 \%$ gel (which was probably more representative of the real-life situation in which these drugs are used and had a patient age range of eight to 103 years) 327 patients experienced 331 adverse events $\{468\}$. Although the majority were 
skin reactions, 24 were related to the gastrointestinal tract. There were eight serious adverse reactions, one of which was "probably" related to topical NSAID use. Unfortunately, this report does not detail the ages of the patients who experienced these adverse effects.

The main source of information regarding adverse drug reactions to topical NSAIDs in the United Kingdom has been data from Yellow Card reports to the $\operatorname{CSM}\{439\}$. These data should be viewed critically as we have no knowledge of the overall numbers of patients exposed to the drugs. However, it is worth noting that up to October 1994, there had been a total of 500 reports of adverse reactions following the topical application of diclofenac, ibuprofen, ketoprofen, piroxicam or felbinac (personal communication, CSM). None of these reactions were fatal, and 54\% were skin or subcutaneous tissue disorders, but over one third of the rest were gastrointestinal effects. It was not possible to determine how many of these reactions were confounded by any additional medication being taken, in particular, oral NSAIDs.

\section{Upper gastrointestinal toxicity}

To assess the independent epidemiological risk of upper gastrointestinal bleeding and perforation associated with topical NSAID use, we have carried out a case-control study in a population of approximately 400,000 people, using the MEMO databases $\{1400\}$. There were 1,103 cases in the study, and the effect of confounding from oral NSAIDs and ulcer healing drugs was removed by conditional logistic regression. The adjusted odds ratios were 1.43 (0.81 - 2.54) and $1.05(0.57-1.92)$ using community and hospital controls respectively. We concluded that the independent risk from topical NSAIDs was minimal. We have since repeated the study in a population of patients over the age of 65 years (unpublished data). We 
should stress that this analysis is not independent as we used a proportion of the cases from the earlier study (a total of 665), but the adjusted odds ratios for the independent effect of topical NSAIDs were slightly higher, $1.74(1.01$ - 3.01) and 1.78 (0.91 - 3.46) using community and hospital controls respectively. This suggests that in an elderly population, topical NSAIDs may carry a small risk of gastrointestinal side-effects.

\section{Renal side-effects}

Up to October 1994, there had been five reports to the CSM of renal dysfunction following use of topical NSAIDs (personal communication, CSM). These were one each of acute renal failure, aggravated chronic renal failure, interstitial nephritis, nephrotic syndrome/nephrosis and urinary incontinence. There have also been case reports in the literature of renal impairment associated with topical NSAID use $\{1112\}\{1017\}$. We conducted a case-control study to address this issue, evaluating the independent effect of topical NSAIDs using 207 cases hospitalised with acute renal failure $\{1401\}$. The odds ratios for ever exposure, adjusted for oral NSAID use, were $1.33(0.79,2.24)$ and $1.04(0.60,1.83)$ using community and hospital controls respectively. We concluded that the independent risk of acute renal failure from topical NSAIDs was minimal. When the analyses were repeated using 139 cases over the age of 65 years (unpublished data), the risks were $1.15(0.61-2.18)$ and $0.60(0.31-1.16)$ and did not suggest that topical NSAIDs carry an independent risk of acute renal failure in the elderly.

\section{Other systemic effects}


There is scarce epidemiological data available regarding the other less commonly occurring adverse drug reactions associated with oral NSAID use, which may also be associated with topical NSAIDs. Up to October 1994, there had only been one case reported to the CSM of abnormal hepatic function following topical NSAID use (personal communication, CSM). There had been a handful of reports of haemopoitetic disorders (one of thrombocytopenia, and two of increased international normalised ratio which may have been the result of interactions), and a slightly higher number of reports of cardiovascular disorders (personal communication, CSM). These included ten reports of peripheral oedema and four of palpitations. In contrast, the number of reports of respiratory disorders is high, 49 in total (personal communication, CSM). These included 19 cases of bronchospasm, 15 of aggravated asthma and ten of breathlessness. However, the problem was specifically highlighted in a CSM publication of December $1989\{599\}$ and it may be that this prompted doctors to report more readily than they would have done so otherwise. In the post-marketing study of felbinac 5\% gel, there was one case of bronchospasm, judged to be "probably" related to topical NSAID use, among 23,590 exposed patients $\{468\}$.

While the overall safety of topical NSAIDs seems reasonable, the data available are incomplete. The main source of information available is spontaneous reporting Yellow Card data $\{327\}$, which is flawed from an epidemiological point of view. The main problems include under-reporting and lack of knowledge regarding the denominator (the total numbers of patients exposed to the drugs) $\{462\}$, doctors having variable reporting thresholds and rates $\{916\}$, and biased reporting and increased reporting following media attention $\{1419\}$. More formal epidemiological evaluation in the post-marketing situation is required to assess the real risks of these drugs. 
There is evidence to suggest that the elderly are at higher risk of drug toxicity $\{661\}\{1771\}$. There is more individual variability in pharmacokinetic and pharmacodynamic response in the elderly $\{660\}$, they usually take more drugs, including over the counter medications, thus increasing the likelihood of interactions $\{660\}$, and compliance may be poor $\{160\}$. The risks from topical NSAIDs are probably dose-related. It may be that the thin epidermal layers of elderly patients have a high rate of absorption. In conclusion, there is little doubt that topical NSAIDs are safer than oral NSAIDs, but they may not be entirely risk-free, and as with all drugs, they should be prescribed with caution in elderly patients. We strongly recommend that more post-marketing surveillance data is accumulated regarding the safety of topical NSAIDs. These should be conducted in as wide a variety of study settings as possible, according to the Safety Assessment of Marketed Medicines Guidelines $\{1533\}$.

\begin{tabular}{lccccccc}
\hline \multicolumn{1}{c}{ Males } & \multicolumn{2}{c}{ Females } & & Total & \\
\hline & Patients & Scripts & Patients & Scripts & Patients & Scripts & Prevalence \\
0-14 yrs & 1,762 & 2,407 & 2,532 & 3,903 & 4,294 & 6,310 & $6 \%$ \\
$15-44$ yrs & 26,523 & 61,273 & 38,310 & 98,964 & 64,833 & 160,237 & $39 \%$ \\
$45-64$ yrs & 21,159 & 94,007 & 28,144 & 161,006 & 49,303 & 255,013 & $56 \%$ \\
$65+$ yrs & 14,236 & 101,285 & 24,846 & 213,164 & 39,082 & 314,449 & $59 \%$ \\
Total & 61,040 & 258,972 & 89,035 & 477,037 & 150,075 & 736,009 & $38 \%$ \\
\hline
\end{tabular}

Table 1 


\begin{tabular}{lccccccc}
\hline & \multicolumn{2}{c}{ Males } & \multicolumn{2}{c}{ Females } & & Total & \\
\hline & Patients & Scripts & Patients & Scripts & Patients & Scripts & Prevalence \\
0-14 yrs & 680 & 741 & 671 & 763 & 1,351 & 1,504 & $2 \%$ \\
15-44 yrs & 4,569 & 5,581 & 6,303 & 8,036 & 10,872 & 13,617 & $7 \%$ \\
45-64 yrs & 4,452 & 7,519 & 7,339 & 13,947 & 11,791 & 21,466 & $13 \%$ \\
$65+$ yrs & 4,545 & 12,019 & 9,818 & 29,855 & 14,363 & 41,874 & $22 \%$ \\
Total & 14,071 & 25,860 & 23,776 & 52,601 & 37,847 & 78,461 & $10 \%$ \\
\hline
\end{tabular}

Table 2 
Legend to Tables

Table 1: Utilisation and prevalence of oral NSAIDs in the Tayside population by age and sex 1989-1993

Table 2: Utilisation and prevalence of topical NSAIDs in the Tayside population by age and sex 1989-1993 